\title{
PRESENCIA DE HUANCAS EN UN SECTOR DE LA CUENCA SUPERIOR DEL RIO HUATATA, CHIARA-AYACUCHO
}

\author{
HÉCTOR CARHUAS TENORIO \\ UniversidAd NACIONAL DE SAN CRISTOBAL DE HUAMAgA \\ hectorcarhuas@hotmail.com
}

\section{RESUMEN}

El presente trabajo es un reporte elaborado a partir de un reconocimiento superficial, en un sector de la cuenca recolectora del rio Huatata. Comprende los territorios de la actual comunidad campesina San Martin de Yanapiruro e Ichubamba, distrito de Chiara, provincia de Huamanga (Ayacucho). Durante el registro superficial identificamos un elevado número de huancas de diferentes formas y tamaños, distribuidas en la cima y laderas de varias colinas entre 3900 a 4300 msnm. A partir del registro arqueológico y descripción detallada examinamos en base a las informaciones etnohistóricas y trabajos referentes al tema para alcanzar nuevos datos a la comunidad en general.

Palabras claves: Huanca, monolito, rio Huatatas, Chiara, Ayacucho, etnohistórica, arqueología, culto al agua, manantial, fertilidad.

\section{ABSTRACT}

This paper is a report from a superficial recognition, in a sector of the dust Huatata River basin. Includes the territories of the current Community campesina San Martin of Yanapiruro and Ichubamba, district of Chiara, province of Huamanga (Ayacucho). During the record surface identified a high number of huancas of different forms and sizes, distributed in the top and slopes of several hills between 3900 to 4300 meters above sea level. From the archaeological record and detailed description we examined on the basis of the information ethnohistorical and works relating to the topic to achieve new data to the community in general.

Keywords: Huanca, monolith, rio Huatatas, Chiara, Ayacucho, ethnohistorical, archaeology, cult of the water, spring, fertility. 


\section{INTRODUCCIÓN}

Este trabajo tiene como base fundamental las exploraciones arqueológicas efectuadas por nosotros durante el año 2015, como parte del curso prácticas pre-profesionales. Llegamos a registrar un total de 16 sitios que contienen numerosas huancas distribuidas de manera misteriosa, en el extremo suprior de la cuenca del río Huatatas. La cantidad de estos monolitos nos lleva a examinar informaciones etnohistóricas de los cronistas y extirpadores de idolatría. Según estos datos en el área andina han sido considerados como ídolos vinculados a fuentes de agua, poblados y terrenos de cultivos. Eran reverenciados y vestidas con ropas de colores denominadas con distintos nombre según en la instancia que se encontraba como "chichic", "guachecol", "chacrayuq" y "marcayuq"(Gentile 2003). Los trabajos arqueológicos son muy escasos, siendo conocidos en la segunda mitad del siglo pasado por Julio C.Tello (1956), quien ha descrito de manera genérica como señales de tumbas y protectores de chacras heredadas. Luego, trabajos de excavación minuciosa han sido efectuados por Bazán del Campo (2007), en el callejón de Huaylas (Ancash), que ha develado ofrendas pertenecientes a distintos periodos prehispánicos debajo de las huancas. Por otro lado, en la costa central han sido vinculados al Precerámico Tardío, por Shady (1999), Falcón 2004 y Ruiz Estrada et al (2011), en los sitios de Caral, Chupacigarro Grande, Caballete, Bandurria, Pampa San José, asociados a plazas y algunos de manera aislados, con posible función astronómica. Por otro lado, para el área del departamento de Ayacucho, se conocen según las referencias de Casafranca (1960) y Lumbreras (1974), una huanca asociada al templo de Chupas de periodo Formativo. Mientras los trabajos exploratorios por parte de la Universidad de Huamanga, registran en su mayoría en la parte alta de los cerros, entre los pisos ecológico suni y Puna. Resaltando abundante huancas en las alturas de Sachabamba (Bautista1993), Chiara (Ortega 2006), Seqchapampa (Roca), Allpachaca y Pariahuanca (Sanchez 2009,2010), Quisuarcancha (De la cruz 2011) y últimamente por nosotros en Ichubamba y Yanapiruro (Carhuas 2015).

\section{ÁrEA DE EsTUDio}

El área de reporte se localiza en la cuenca superior del r\{io Huatata, uno de los afluentes del rio Huarpa, ubicado al sureste de la actual ciudad de Ayacucho, sierra sur central del Perú. Específicamente comprende los territorios del actual comunidad campesina San Martín de Yanapiruro e Ichubamba, distrito de Chiara, provincia de Huamanga, región Ayacucho. La configuración topográfica según la descripción de León Nina (2010), es de origen glaciar que presenta montañas plegadas, abras, afloramientos rocosos, laderas empinadas y colinas ovaladas llegando a una altitud 3017 m.s.n.m(Cerro Chaupi Urqu). Presenta abundantes fuentes de agua que originan a las quebradas Cayramayu, Rudeohuayqu y Hatun Sallahuayqu, afluentes principales del río Huatatas. El entorno ecológico, según las propuestas de Javier pulgar Vidal (1996), abarca dos regiones: Suni (3500 a 4000 msnm) y Puna ( 4000 a $4800 \mathrm{msnm}$ ), donde la vegetación predominante es ichu (stypha ichu), cubriendo casi en su totalidad. También son habitat de animales silvestres como la vizcacha (lagidium peruanun), vicuña (Vicugna vicugna), zorro (Canis calpocus) y aves silvestres como perdiz (Nothroprocta pantlandi), etc. El clima es seco donde la presencia de lluvias distinguen dos estaciones bien marcadas: la época de lluvias con precipitaciones entre enero y marzo; y la época de seca de mayo a octubre, en que la aridez es muy intensa y con heladas frecuentes, aunque en algunos casos se da lluvias anormales. 


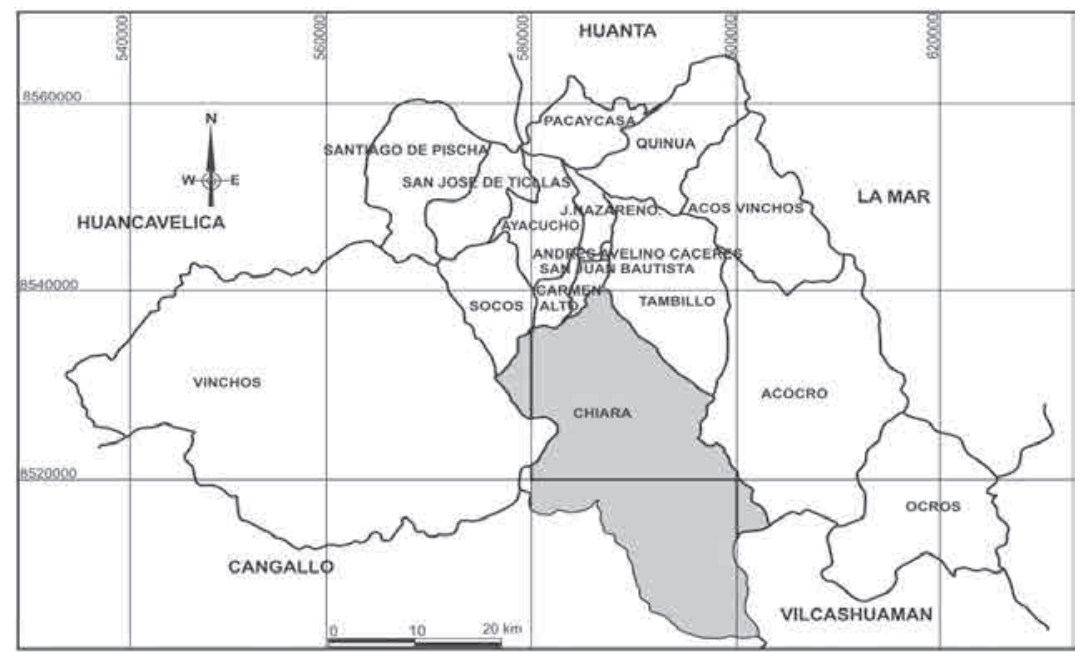

Figura 1. Ubicacion del área de estudio dentro de la mapa política de la provincia de Huamanga, Ayacucho.

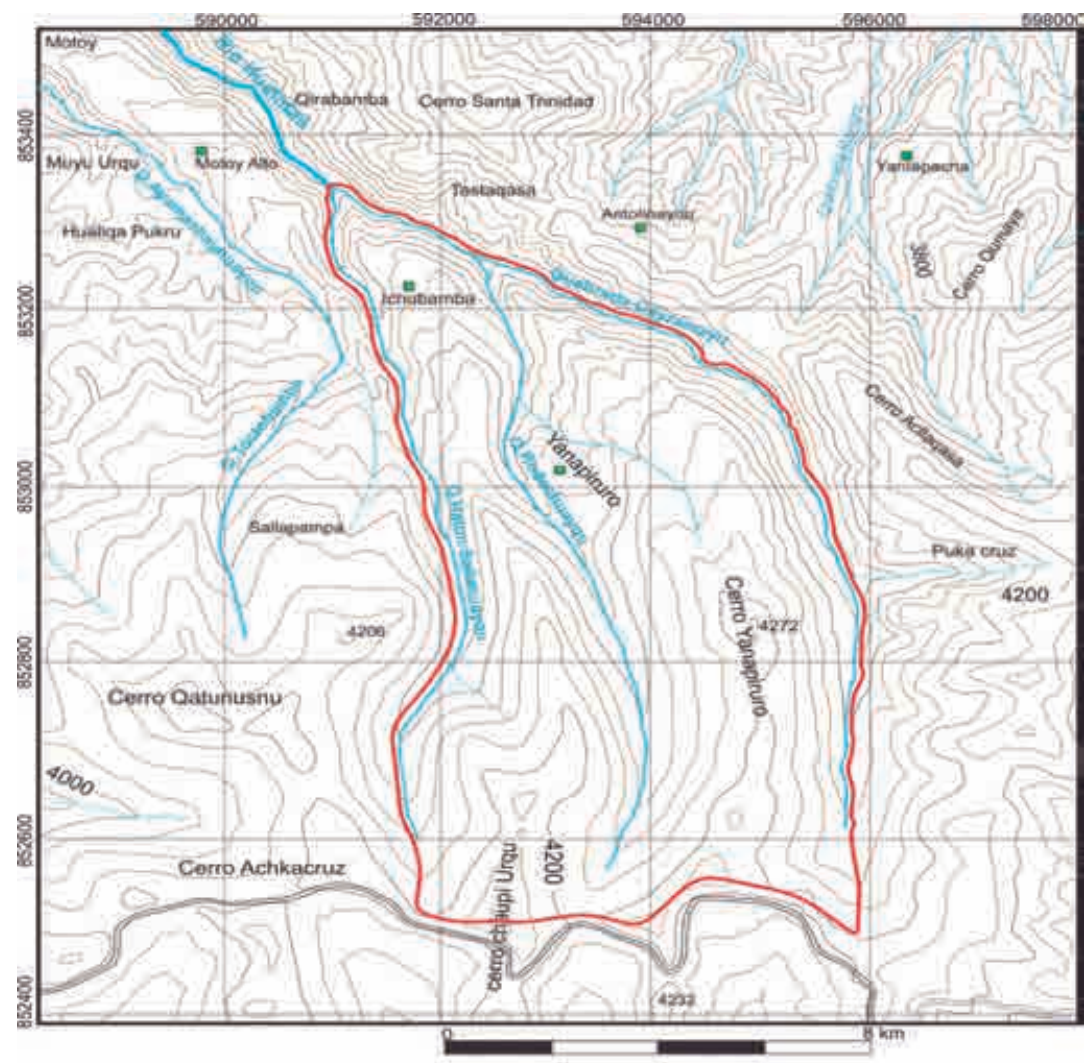

Figura 2. Ubicación del área de estudio, dentro del mapa hidrográfico de la cuenca alta de río Huatata. 


\section{Metodología y Técnicas del Registro}

Durante el proceso de trabajo de investigación, hemos empleado los siguientes pasos: para la ubicación y delimitación del área de estudio, se recorrió a la carta nacional del Instituto Geográfico Nacional del Perú, en la escala 1:100 000, de la hoja 27-ñ, que corresponde al territorio de la provincia de Huamanga y tomamos como puntos las quebradas de Cayramayu y Hatunsallahuayqu,cuenca superior del río Huatata. Asimismo, hemos empleado mapas políticos a nivel regional, provincial, distrital y de la comunidad perteneciente. El registro realizamos de manera directa, con un recorrido a pie en transectos paralelos (trayectorias lineales) y en algunas ocasiones onduladas, adecuándose a la configuración topográfica y también nos facilitó los datos proporcionados de los pobladores de la zona. El registro ha comprendido la documentación escrita, gráfica y fotográfica, siendo el escrito en el cuaderno de campo y fichas impresas, ubicando con el GPS las coordenadas, la altitud y los aspectos característicos de cada huanca en situ. Finalmente, hemos realizado la sistematización empleando el material bibliográfico con la comparación y análisis de los datos obtenidos en el campo, como en el gabinete.

\section{Datos Etnohistóricos Referente al Tema}

Las referencias acerca de los monolitos huancas son proporcionadas por los cronistas y extirpadores de idolatría durante las primeras épocas de la Colonia. Uno de los sacerdotes que ha viajado por la gran mayor parte del Perú en el siglo XVI fue Cristóbal de Albornoz, quien ha registrado numerosos ídolos de piedra que eran reverenciados en diferentes pueblos andinos. Al llegar por la zona de Ayacucho identifica en los cerros Osqonta, Parinacocha y Sarasara, que según sus propias palabras: "«Auqui uscuntay, guaca de los indios soras, era una piedra en un cerro que se llama ansi.[...] Chuchuranac es un cerro junto a parinococha....Era una piedra vestida en una punilla cerca del cerro nevado. Sarasara es un cerro nevado y en el esta una piedra del dicho nombre....visitando esta provincia, halle muy much a suma de guacas y las destrui y queme.....(Albornoz 1984[1584]:207).Numerosos ídolos de piedra existieron en las cumbres de los cerros, las cuales han sido desaparecidas durante la evangelización cristiana, pero mucho de ellos quedaron como un testimonio de una gran religiosidad andina. De manera similar Francisco de Ávila para el caso de Huarochirí, recoge información relacionado al agua:“....los habitantes de este ayllu, limpian el acueducto conforme lo hacían en la antigüedad, en el mes de mayo. En esa ocasión todos, toda la gente, iba hasta la piedra en que se convirtió Chuquisuso. Llevaban chicha, una clase de comida que se llama ticti y cuyes y llamas para adorar a esa mujer demonio."(Ávila 1966 1598:53). Según esta información la veneración y las ofrendas a un ídolo de piedra, eran importante en cada limpieza del canal de agua. En su tradición y concepción de este pueblo, Chuquisuso era una mujer petrificada a falta de agua y luego fue sacralizada y venerada en fechas importantes. Por otro lado el padre jesuita José de Arriaga argumenta:“...algunas piedras muy grandes también adoran y mochan, y les llaman con nombres particulares y tienen sobre ellos mil fabulas de conversiones y metamorfosis y que fueron antes hombres que se convirtieron en aquellas piedras.... Chichic o huanca llaman una piedra larga que suelen poner empinada en sus Chacaras, y la llaman tambien Chacrayoc, que es el senor de la chacara, porque piensan que aquella chacara fue de aquella huaca, y que tiene a cargo su aumento, y como a tal la reverencian, y especialmente en tiempo de las sementeras le ofrecen sus sacrificios"(Arriaga 1968(1621) Capitulo II).

La información de Arriaga sintetiza y nos brinda una serie de informaciones producto de la extirpación de idolatría, en donde los personajes convertidos en piedras eran denominados "huanca" o 
ficios. Asimismo, hay otra información similar al de Ávila, que fue recogido por Pierre Duviols (1973), del Archivo Arzobispal de Lima, un ídolo de piedra relacionado a fuente de agua: “....sumac guanca que esta junto a la cabesera de un puquio que disen es dueño del dicho puquio y que primero fue yndio llamado Tupin Guailca,el qual dicen y tienen tradición crio aquel puquio"(Duviols 1973:163). Hay una numerosidad de fuentes escritas de origen colonial que contienen datos importantes, mucho de ellos con un contenido mitohistórico, que para muchos de los investigadores no es confiable. Nosotros rescatamos como dice María Rostworoswski (1999),que estas narraciones míticas, contienen un fondo de sucesos verídicos que nos pueda dar algún alcance importante.

\section{Registros de Sitios con Huancas en un Sector de la Cuenca Superior de Rio Huatata}

A continuación se describen los 16 sitios arqueológicos ubicados en la cima y ladera de los cerros, en los sectores de Ichubamba y Yanapiruro, en la cuenca recolectora del río Huatata.

REMILLA URQUN. Se localiza en un promontorio con abundante pedregal, en las coordenadas de UTM: 18L 0592019 E, $8529736 \mathrm{~N}$, a una altitud de $3944 \mathrm{msnm}$, sector Ichubamba. Conformado por 2 huancas uno de ellos se ubica en el extremo superior de la cima, con una altura de $60 \mathrm{~cm}$ y está alineada con piedras medianas a un agujero de forma circular con característica de una cista. Asimismo hacia la ladera oeste se ubica una Huanca de $1.60 \mathrm{~m}$ de altura, rodeada con piedras medianas y presenta alineamiento de piedras, con remoción actual producto del huaqueo.

SUAPA TIYANAN. Se ubica en la cima y ladera de una colina, al lado sur del promontorio de Remilla Urqun, en las coordenadas UTM: 18L $0592295 \mathrm{E}, 8528970 \mathrm{~N}$, a una altitud de $4048 \mathrm{msnm}$, sector Ichubamba. Está conformado por 12 huancas distribuidas tanto en la cima y ladera del cerro mencionado. Los más resaltantes son, dos corrales de forma semicirculares que uno de ellos contiene en el centro dos huancas de $70 \mathrm{~cm}$ y $1.30 \mathrm{~m}$ alterada por el huaqueo contemporáneo. En las proximidades se ubican los demás monolitos de morfología variada que alcanzan hasta $2 \mathrm{~m}$ de altura, algunas individuales y otras en dualidad, en el medio de círculo de piedras de 2 metros de diámetro. Además asociado a este sitio se halló fragmento de cerámica no diagnostica todas erosionada.

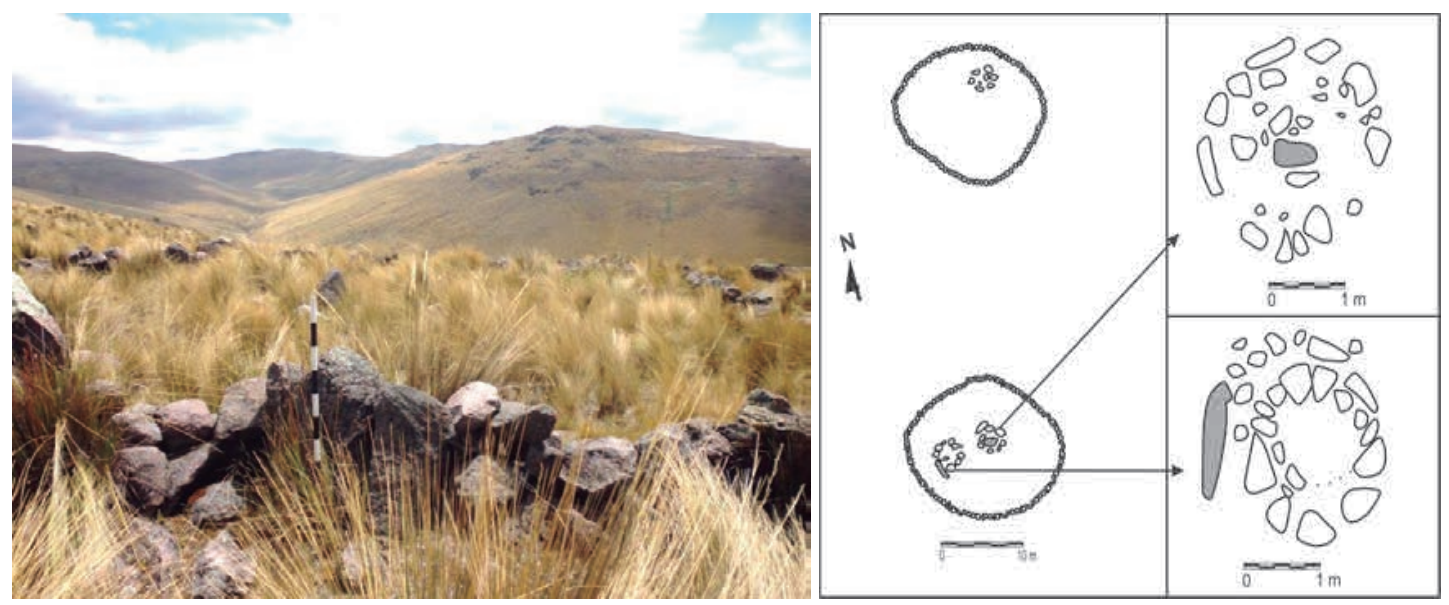

Figura 3. Vista en detalle del corral con huanca y croquis de localizacion en el sitio de Suapa Tiyanan, sector Ichubamba. 
URQUPA SIMIN .Ubicado en la cima de una colina, al sur del sitio Suapa Tiyanan, en las coordenadas UTM: 18L 0592757 E, 8527624 N, a una altitud de 4190 msnm, en el sector de Ichubamba. Está conformado por 18 huancas distribuidas en la cima y laderas del cerro a distancias variadas. Se encuentran dispuestas en posición vertical y algunas inclinadas sobre una pequeña plataforma circular de 2.50 a $3 \mathrm{~m}$ de diámetro. Predominan las huancas individuales, en dualidad y en trio que varían en la longitud de $90 \mathrm{~cm}$ a $1.70 \mathrm{~m}$, generalmente unidas por dos muros de una sola hilada en paralela. Asimismo, hemos identificado un corral pequeño de forma circular con 8 metros de diámetro, que contiene en la parte céntrica una huanca de $1.20 \mathrm{~m}$ de longitud, fuera de su contexto original. La morfología es de aspecto tubular, rectangular, lanceolada e irregular y la cultura material asociado son fragmento de cerámica de acabado llano erosionado. Esta zona hasta la actualidad es respetada y visto como un lugar sagrado donde los pobladores efectúan sus pagos relacionados a la fertilidad de los ganados.
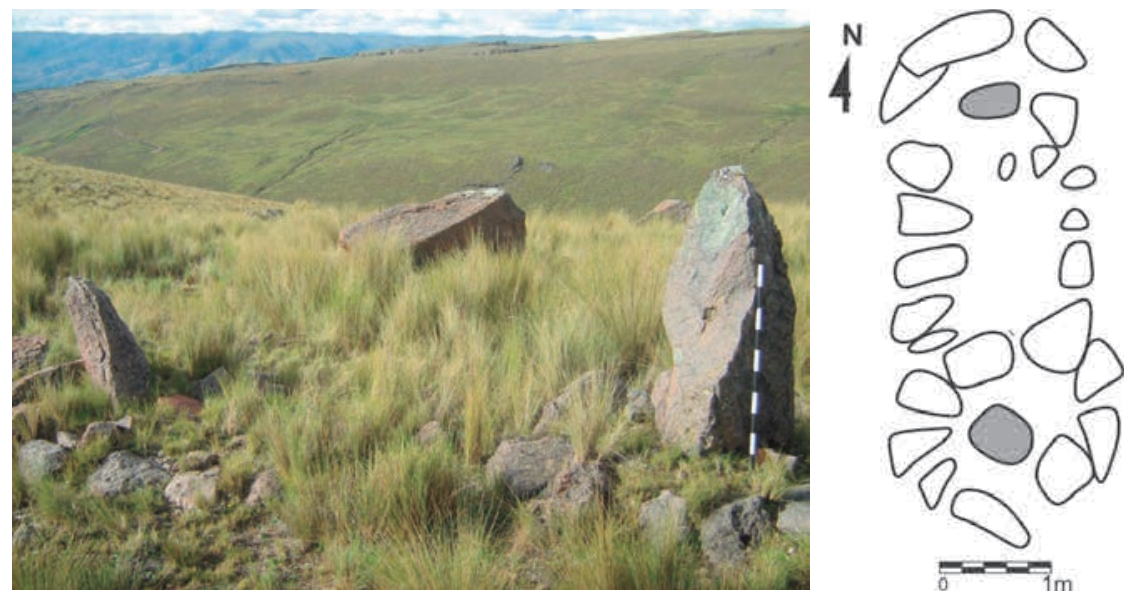

Figura 4.Vista en detalle de la huanca en dualidad en el sitio de Urqupa Simin, sector Ichubamba.

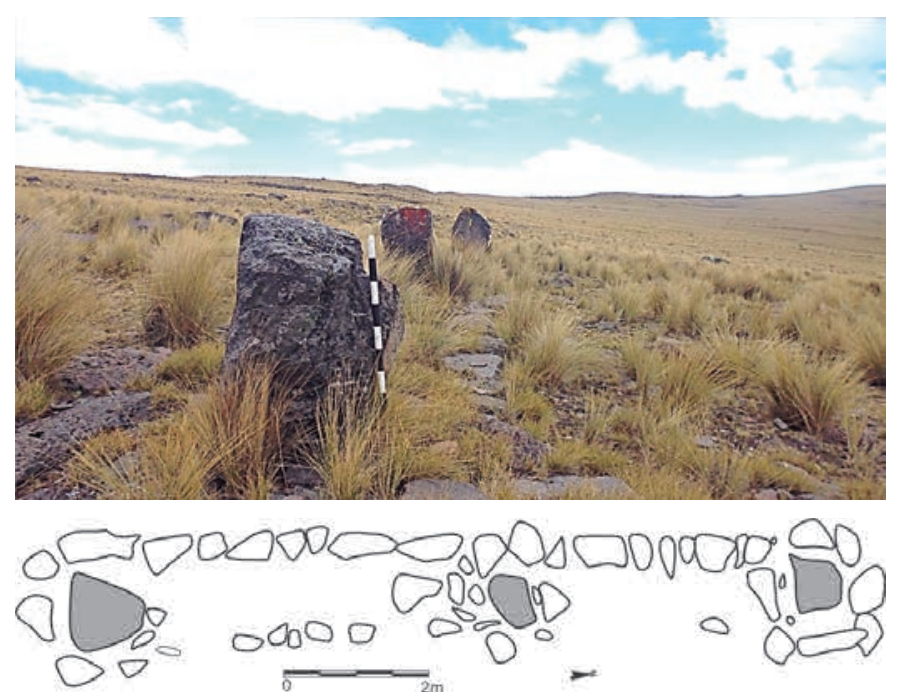

Figura 5. Detalle de las tres huancas alineadas en el medio de una plataforma de piedras en el sitio de Urqupa Simin, sector Ichubamba. 
AQCHIPA WACHANAN. Se ubica al lado sureste del cerro Urqupa Simin, en las coordenadas de UTM: 18L 0592993 E, $8526998 \mathrm{~N}$, a una altitud de $4171 \mathrm{msnm}$, en el sector de Ichubamba. Conformado por un total de 8 huancas distribuidas de manera individual en la ladera del cerro. Estos monolitos se encuentran dispuestos en posición vertical sobre una pequeña plataforma semicircular y en algunas ocasiones hay amontonamiento de piedras a manera de sostener. El tamaño oscila desde $50 \mathrm{~cm}$ a 2 metros de altura y la morfología es generalmente rectangular, lanceolada e irregular. Tres de las huancas se ubican en el medio de pedregales y asociado a un corral antiguo; mientras una huanca está muy próxima a un manantial.

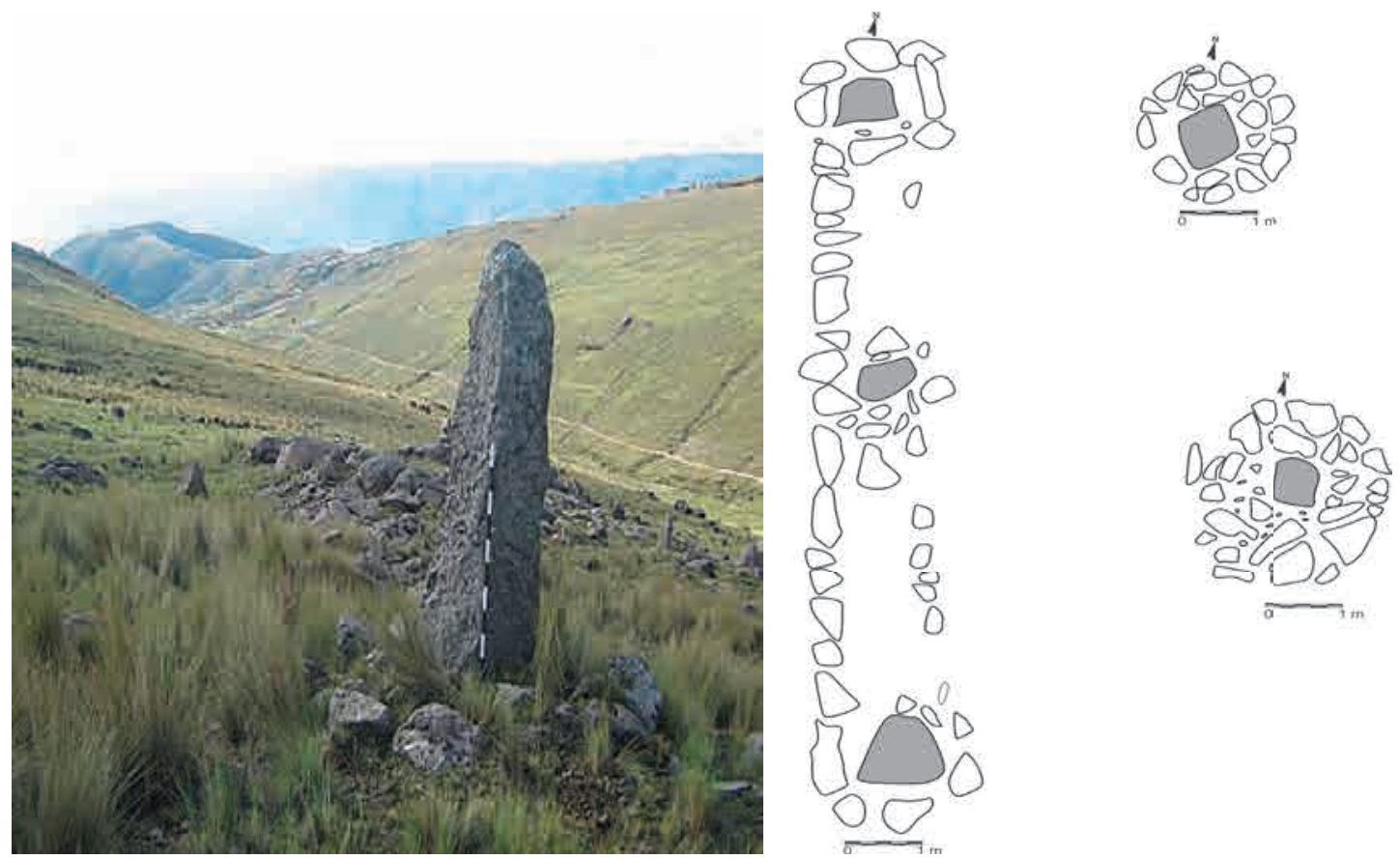

Figura 6. Huanca individual de forma rectangular en el medio de círculo de piedras en el sitio de Aqchipa Wachanan, sector Ichubamba.

AYARANRA URQU. Ubicado en una colina próxima a las llanuras de Platu Qasa, en las coordenadas de UTM: 18L 0593059 E, $8526370 \mathrm{~N}$, a una altitud de $4215 \mathrm{msnm}$, en el sector de Ichubamba. Se tratan de un total de 8 huancas que se encuentran dispuestas de manera vertical y algunas inclinadas en el medio de una pequeña plataforma de piedras de diferentes tamaños. La distancia de su ubicación es variable estando 5 monolitos de manera individual y 3 juntos unidas por dos muros de una sola hilada en paralelas, siendo el más resaltante con 2.80 metros de altura y un aspecto zoomorfo en el extremo superior. Las demás monolitos son de diferentes tamaños que varían de $60 \mathrm{~cm}$ a $2.20 \mathrm{~m}$ de altura y tienen forma alargadas e irregulares.

LUCAPA TUMANAN. Se ubica en una zona ligeramente explanada, cerca de un conjunto de manantiales, en las coordenadas de UTM: 8526038 N, 18L 0592742 E, a una altitud de 4206 m.s.n.m.,en la jurisdicción del actual poblado de Ichubamba. Está conformado por 6 huancas que se encuentran en posición vertical e inclinada distribuidas en diferentes partes de la planicie y todas están sobre un 

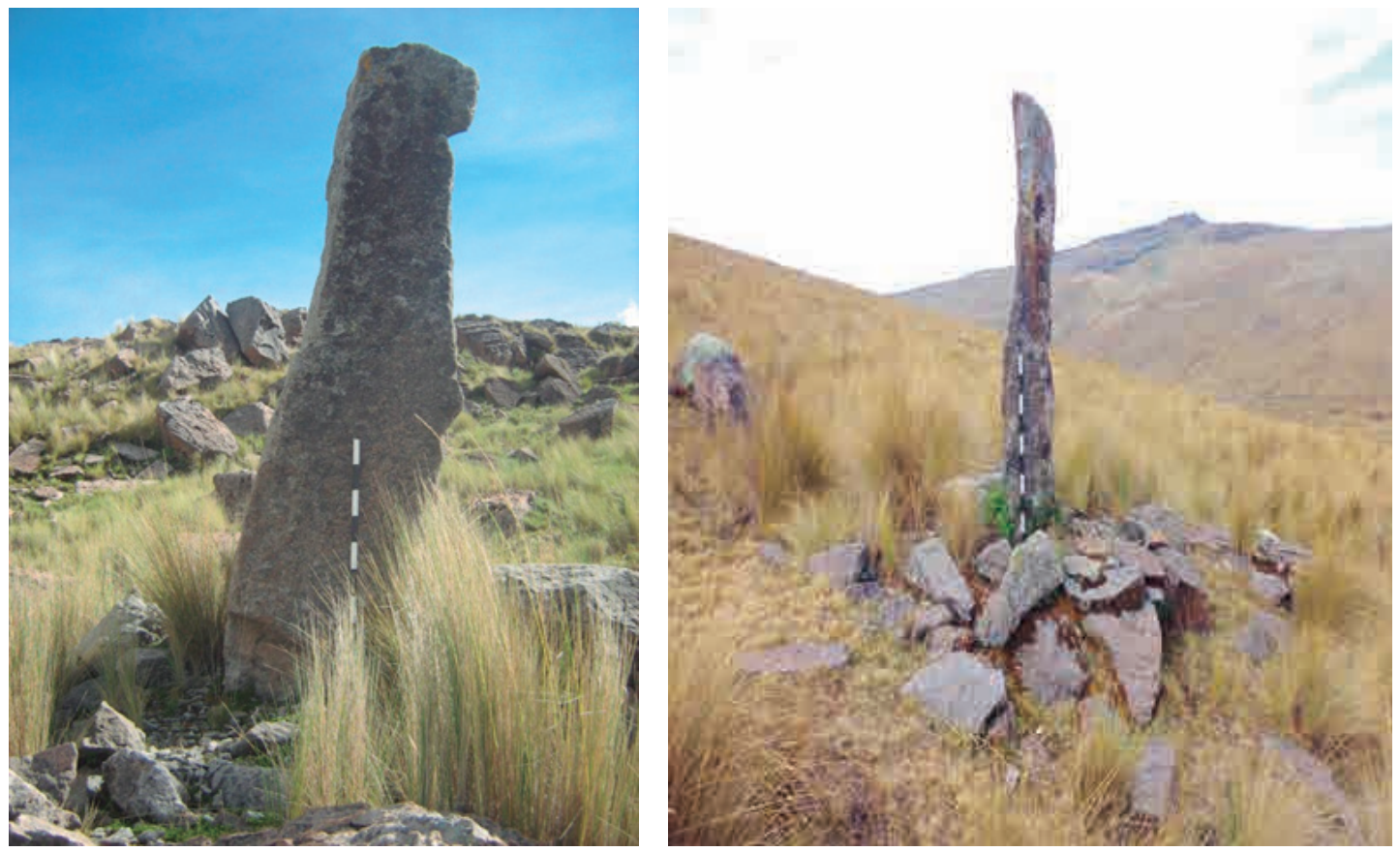

Figura 7.Vista en detalle de las huancas que alcanzan a dos metros de altura en el sitio de Ayaranra, sector Ichubamba.

soporte de piedras que forman semicircular. La morfología generalmente es irregular con las medidas que oscilan de $50 \mathrm{~cm}$ a $1 \mathrm{~m}$ de longitud. Dos huancas individuales se encuentran sobre un suelo rocoso con escasa vegetación; asimismo la huanca en dualidad está asociado a un manantial y dos están aislados unidas por dos muros de una sola hilada en paralela.

HATUSNIYUQ. Se localiza al lado oeste del sitio Lucapa Tumanan, en las coordenadas de UTM: 18L $0592041 \mathrm{E}, 8526944 \mathrm{~N}$, a una altitud de $4111 \mathrm{msnm}$, en el sector de Ichubamba. Está conformado por 8 huancas que se encuentran en posición vertical y algunas en horizontal producto del huaqueo contemporáneo. La morfología es variada, siendo irregulares, rectangular y lanceoladas, con una longitud que oscila desde 1.20 a 2 metros de altura, en el medio de círculo de piedras de 2 a 3 metros de diámetro. La gran mayoría se distribuye de manera individual sobre una roca natural y abundante pedregal; mientras una huanca muy próximo a un manantial y dos huancas juntas unidas por dos muros paralelos de una sola hilada.

HERRASRUWANA PAMPA. Ubicado al lado norte del cerro Chaupi Urqu, cerca de la línea de gaseoducto de Camisea, en las coordenadas de UTM: 18L $0592363 \mathrm{E}, 8525736 \mathrm{~N}$, a una altitud de 4221 msnm, en el sector de Ichubamba. Se trata de cinco corrales antiguos de formas rectangulares y ovaladas que una de ellas presenta en el centro una huanca de $1.20 \mathrm{~m}$, en posición vertical rodeado con alineamiento de piedras pequeñas. No hay indicio de otro tipo de material cultural y en los alrededores existen bofedales y manantiales que dan origen a la quebrada Hatunsallahuaycco.

CHAUPI URQUE. Es un cerro de mayor elevación próximo a la carretera asfaltada Ayacucho-Andahuaylas, ubicado en las coordenadas de UTM: 18L $0592011 \mathrm{E}, 8524662 \mathrm{~N}$, a una altitud de $4317 \mathrm{msnm}$, 
en el sector de Ichubamba. En la ladera superior de este cerro se encuentran distribuidas un total de 10 huancas de diferentes formas y tamaños en posición vertical y algunas ligeramente inclinadas. Algunas se encuentran de manera individual y otras en asociación hasta cuatro monolitos juntas con una longitud que varía de $50 \mathrm{~cm}$ a 2 metros, sobre un soporte de piedra de forma circular. Generalmente están sobre una roca natural y abundante pedregal. Lo más sobresaliente es una huanca con evidencia de pintura rojo ocre en la parte plana, con líneas delgadas verticales producto de algún tipo de ritual. En cuanto a material cultural, se halló fragmentos de cerámica de tamaño miniatura con un acabado burdo erosionado, la cual no permite la asociación cronológica.
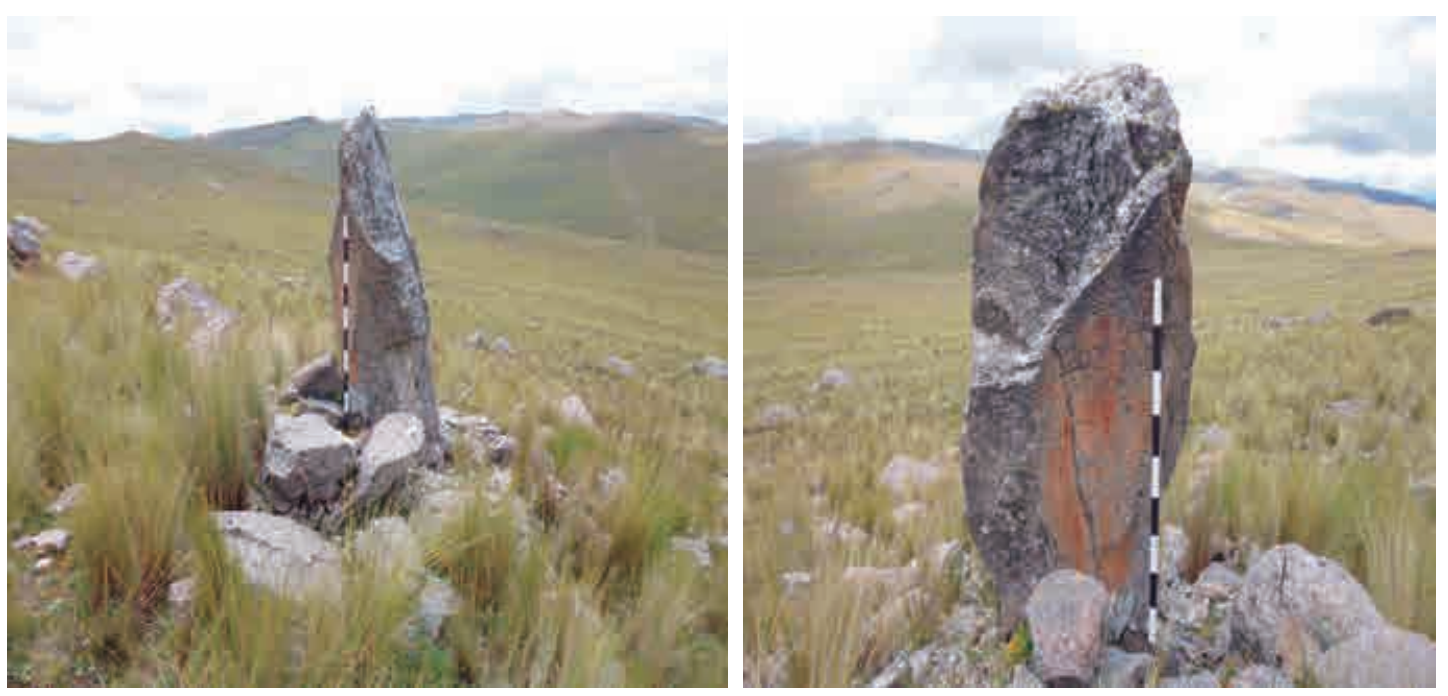

Figura 8. Huanca de forma irregular con evidencia de pintura rojo ocre, ubicado en el cerro Chaupi Urqu, sector Ichubamba.

QAYAKUQ MACHAY QASA. Se encuentra ubicado en una abra, al norte del cerro Yanapiruro, en las coordenadas de UTM: 18L 0594335 E, 8530028 N, a una altitud de 4095 msnm, en el sector de Yanapiruro. Conformado por 2 huancas; siendo la primera de forma lanceolada, con una longitud de $1 \mathrm{~m}$, ubicado en el medio de un corral circular de $8 \mathrm{~m}$ de diámetro, removida de su posición original producto del huaqueo contemporáneo. A pocos metros del corral se ubica una huanca de forma irregular, con una longitud de 1.20 metros, dispuesta en posición vertical sobre un soporte de piedras hecha de forma circular de $2 \mathrm{~m}$ de diámetro. En los alrededores se aprecian alineamientos de piedras alteradas por las obras públicas.

PUKAQAQA .Se encuentra ubicado próximo al cerro Yanapiruro, en las coordenadas de UTM: 18L 0594684 E; 8528710 N, a una altitud de 4162 msnm, en el sector de Yanapiruro. Se tratan de 4 huancas individuales, distribuidas a diferentes distancias en la ladera del cerro; cuya altura varía de $90 \mathrm{~cm}$ a 1.70 metros y tienen formas lanceoladas e irregulares. Dos de los monolitos están muy próximos a un camino actual ligeramente inclinadas; mientas dos huancas se encuentran de manera individual en el medio de abundante roquedal.

CERRO YANAPIRURO. Es una de las colinas que termina en una plataforma natural de rocas, ubicado en las coordenadas de UTM: 18L 0594624 E, 8507580 N, a una altitud de 4272 msnm. En la parte 
lateral sur se encuentra una huanca de forma alargada, de 1.80 metros de longitud en el medio de la cantera de andesita. Está dispuesta en posición vertical rodeada con piedras planas, sin ninguna asociación de otro elemento cultural.

SUYTU RUMI QATA. Se ubica al lado suroeste del cerro Yanapiruro, en una ladera con abundante pedregal, en las coordenadas de UTM: 18L 0509372 E, $8526232 \mathrm{~N}$, a una altitud de $4186 \mathrm{msnm}$, sector de Yanapiruro. Se trata de un total de 8 huancas dispuestas en posición vertical y algunas ligeramente inclinadas, sobre una pequeña plataforma semicircular de piedras pequeñas de 2 a 4 metros de diámetro. Estas huancas se caracterizan por estar de manera solitaria y algunos en dualidad, unidas por dos muros de una sola hilada en paralela. Las medidas varía desde $50 \mathrm{~cm}$ a 3.50 metro de longitud, con una morfología rectangular, irregular y el más grande es ornitomorfa, siendo el más resaltante de todas las huancas registradas. En las proximidades al lado oeste, se encuentran pequeños manantiales y abundante pedregal.

PUTAQA. Se localiza en el extremo superior del río Cayramayu, en una zona con abundantes manantiales y pedregal, en las coordenadas de UTM: 18L $0594701 \mathrm{E}, 8525564 \mathrm{~N}$, a una altitud de 4203 msnm, en el sector de Yanapiruro. Conformado por un total de 7 huancas que se encuentran distribuidas sobre un promontorio con abundante pedregal. Todas están individualmente dispuestas en posición vertical y algunas ligeramente inclinadas, en el medio de una especie de estructura de forma semicircular de 2.50 a 3 metros de diámetro. La longitud varía desde 1 a 2.40 metros y asimismo la morfología es tubular, rectangular, lanceolada e irregular y la distancia de una huanca a otra es variable. Además en los alrededores de las huancas se ha evidenciado fragmentos de cerámica en escasa cantidad todas erosionadas.

PUTAQA QASA. Se localiza en el extremo sureste del área de estudio, próximo a la carretera asfaltada Ayacucho- Andahuaylas, en las coordenadas de UTM: 18L 0594812 E, 8524974 N, a una altitud de $4206 \mathrm{msnm}$, en el sector de Yanapiruro. Es un corral antiguo de forma ovalada de $11 \mathrm{~m}$ de diámetro y en la parte central se encuentra una huanca en posición vertical de $50 \mathrm{~cm}$ de longitud, de morfología tubular rodeado con piedras pequeñas. Adosados a este corral se encuentran tres pequeños recintos semicirculares con 3 metros de diámetro cubiertas con vegetación de gramínea y en las proximidades asociado a un camino antiguo se encuentra una apacheta.

LAYQA QASA. Es una colina ubicada próxima a la carretera asfaltada Ayacucho-Andahuaylas, en las coordenadas de UTM: 18L 0593665 E, 8524620 N, a una altitud de $4291 \mathrm{msnm}$, en el sector de Yanapiruro. Se trata de un total de 12 huancas distribuidas tanto en la cima y ladera de esta colina. Tres de las huancas se ubican juntas en el extremo superior de la cima; asimismo tres huancas de tamaños diferenciados en la ladera oeste rodeados con piedras medianas de forma semicircular de $2.50 \mathrm{~m}$ de diámetro. Por otro lado, dos de las huancas una vertical y la otra horizontal se encuentran juntas unidas por dos muros de una sola hilera en paralela. Por último, los 4 monolitos restantes se encuentran de manera solitaria en diferentes distancias asociado a afloramiento de roca natural. Las medidas de estos monolitos son variados desde $50 \mathrm{~cm}$ a 2 metros de longitud y asimismo la morfología es tanto tubular, rectangular, lanceolada e algunos irregulares.

\section{COMENTARIO}

La numerosidad de Huancas registradas en nuestra área de estudio (108 monolitos), es una de las evidencias arqueológicas complejas por su ubicación cronológica y las características que presenta en el tamaño y forma. 


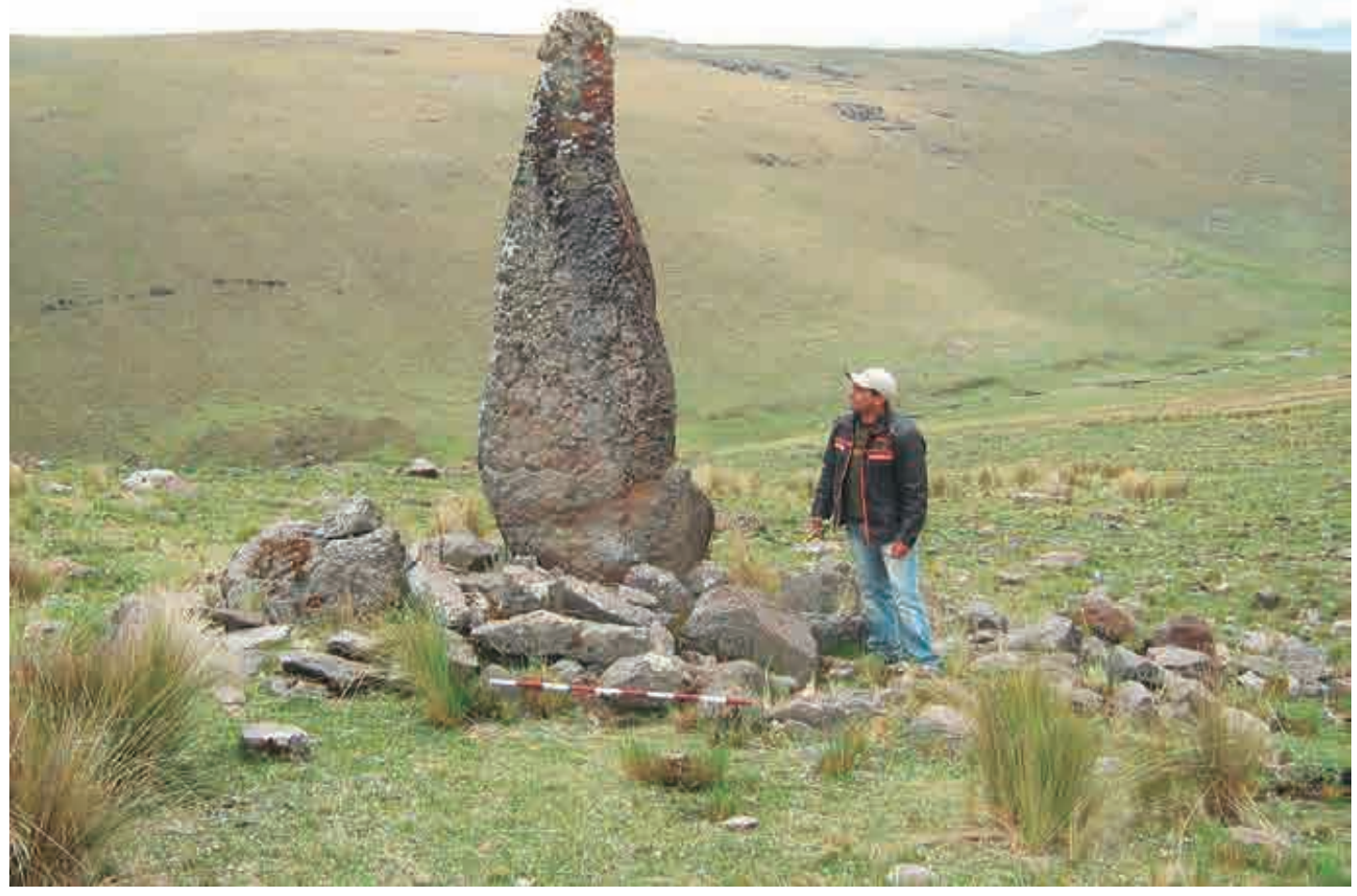

Figura 9. Huanca de mas de tres metros de altura,en Suyturumiqata,sector Yanapiruro.
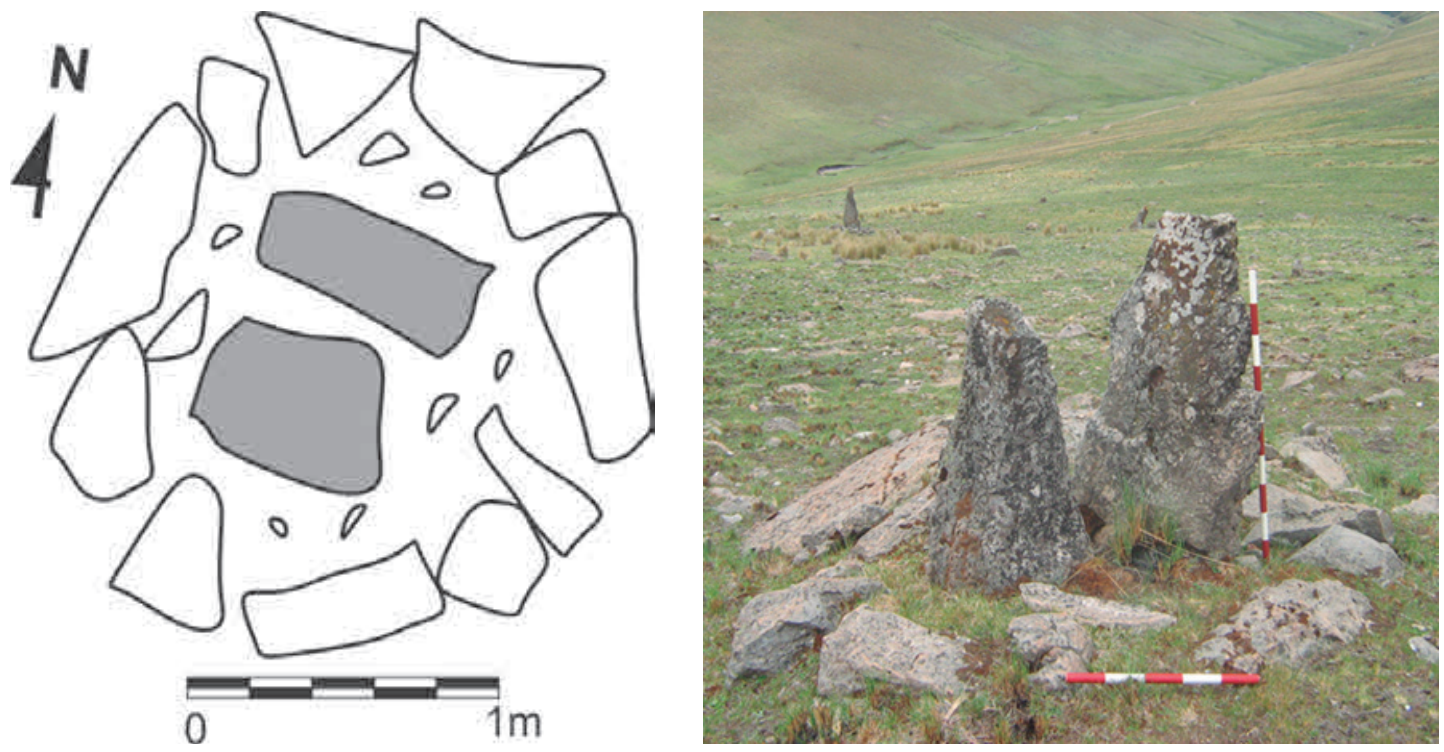

Figura 10. Huanca en dualidad dispuestas en el medio de círculo de piedras, en el sitio de Suyturumiqata, sector Yanapiruro. 

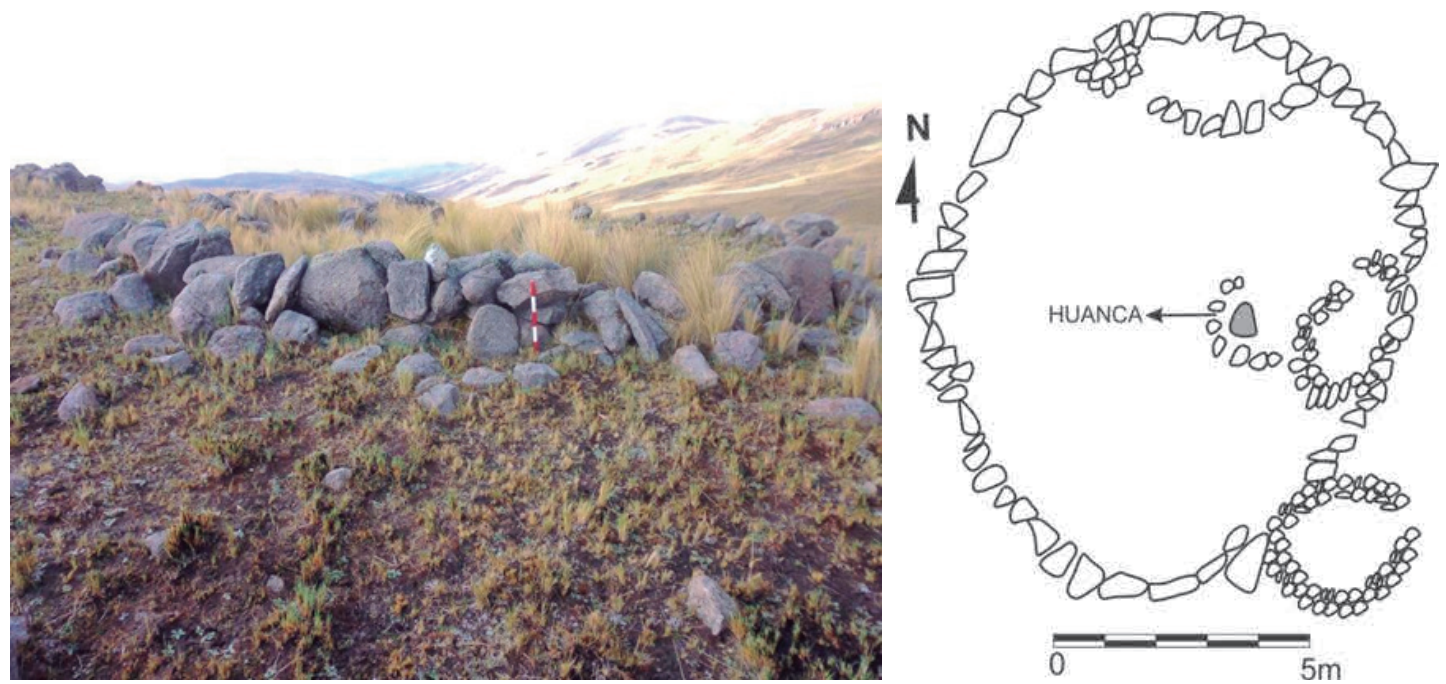

Figura 11. Dibujo de planta del corral de forma ovalada, asociado a huanca y recintos circulares, en el sitio de Putaqa qasa, sector de Yanapiruro.
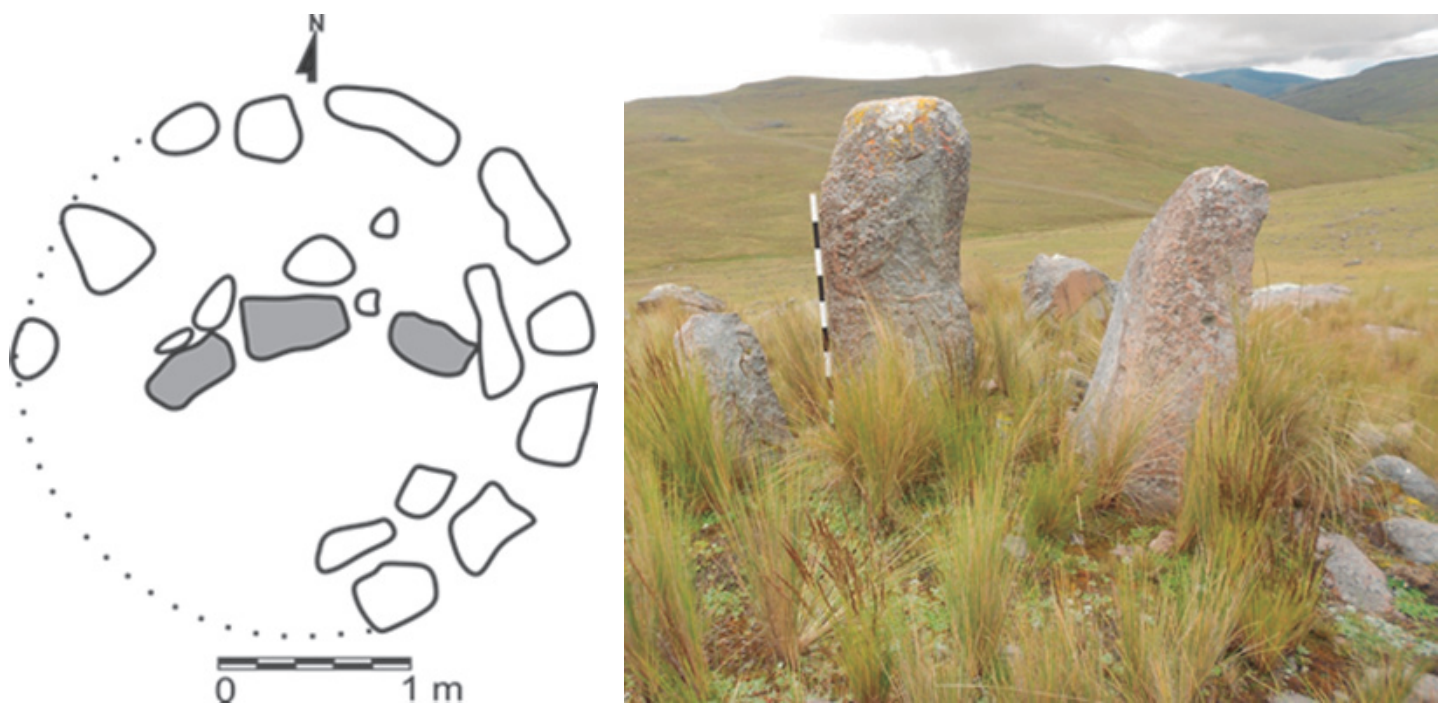

Figura 12. Huanca en trío sobre un soporte de piedras semicircular, en la ladera del cerro Layqa Qasa, sector Yanapiruro.

En el área de los Andes Centrales los últimos reportes arqueológicos ubican, en los sitios correspondientes desde el Precerámico Tardío hasta el Horizonte Tardío. Por otro lado, en las fuentes etnohistóricas están relacionados a mitos y leyendas que contienen acontecimientos trascendentales e extraordinarios. El mito de Viracocha (Pease 1973),Vichama (Ruiz et al 2011), origen de los incas (Rostworowski 1999), entre otros, contienen petrificación de personajes importantes. En la actualidad perduran todavía relatos míticos, donde aparecen personas trasformadas en piedras con la in- 
tervención divina, como en el mito fundacional de un pueblo en la cuenca de río Pampas, Ayacucho (Maldonado 2013). En la mitología andina prehispánica la conversión en piedra no significaba muerte, más bien era la peremnización y sacralización en un símbolo fertilizador y protector que conecta con los tres espacios, el mundo de abajo (uku pacha), el mundo de intermedio (kay pacha) y el mundo de arriba (hanaq pacha). Además, las huancas se ubicaban en lugares importantes por los hechos del antepasado y estar ligadas a la fecundidad, siendo consideradas como fundadores de pueblos, dueños de chacras, acequias y puquios (Duviols 1973:163). De esta manera el huanca no solo es simple piedra sino, al ser venerado se convierte en un ícono que tiene varios significados y estos significados están siempre presentes en los símbolos y son dramatizados en los mitos cobrando sentido sobre la forma de vida y la manera de comportamiento en el mundo (Gertz 2003).

Las huancas que corresponden a nuestro registro, según la ubicación espacial están localizadas en una zona donde se originan un conjunto de riachuelos y quebradas, algunos de los monolitos asociados directamente a manantiales como en el sitio de Aqchipa Wachanan, Lucapa Tumanan y Qatusniyuq. Este caso es algo similar en otras partes del área andina, como en Canta, Lima, alrededor del canal de agua (Farfan 2002), en el distrito de Shupluy del departamento de Ancash, cerca de una laguna (Del Campo 2007) y en las cercanías de nuestra área de estudio en Quisuarcancha próximo a un manantial (De la Cruz 2011). Estos datos nos permiten acercarnos a las informaciones coloniales de carácter etnohistóricas como el de Ávila (1966[1598]), una mujer petrificada a causa de la sequía y luego es sacralizada y venerada en cada limpieza del canal de agua. La escases del agua y presencia de sequias han sido uno de los problemas más graves que se ha presentado en la historia de la humanidad y hay referencias de cambios climáticos en los diferentes periodos de la época prehispánica.

Esto nos da a entender y reafirmar hipótesis de nuestro trabajo (Carhuas 2015), que las numerosas huancas existentes en la cuenca superior del río Huatata probablemente tenga una explicación mitológica vinculada a culto al agua. El origen de los ríos fueron los puntos claves para las ceremonias y rituales, cuyas evidencias de pintura rojo ocre hemos identificado en una de las huancas de Chaupi Urqu. Asimismo los que están en zonas infértiles sobre la roca natural y abundante pedregal también lo mismo estaría cumpliendo, como símbolo fertilizador. Además, es posible que la numerosidad de Huancas registradas al sur de la provincia de Huamanga por Bautista (1993), Ortega (2006), Roca (2007) y Sánchez $(2009,2010)$, estuvieran relacionados a este tipo de ceremonias, pues según la distribución espacial están próximos a las nacientes de los ríos. Asimismo, es evidente que las punas de Toccto, cerca de nuestra área de estudio, es una franja montañosa interfluvio donde existen una multitud de huancas, convirtiendo en un paisaje ritual, que con la llegada de los incas ha continuado cuya evidencias es el Usnu (Vivanco y Meddens 2010; Cavero 2010), asociado a Sayhuas y pagapus contemporáneos. El agua como bien se sabe es un elemento vital que recibió culto desde los tiempos prehispánicos y actualmente perdura en muchas comunidades con una variedad de festividades en relación a la fertilidad y abundancia. Finalmente quisiéramos alcanzar Huancas asociadas a corrales antiguos ubicados en la parte céntrica, las cuales corresponden a los sitios de Qayakuqmachay Qasa, Putaqa Qasa, Herrasruwana, Urqupa Simin y Suapa Tiyanan, todos tienen las mismas características ubicados por Ortega (2006), en el sitio de Altarpata, próximo a nuestra área de estudio. Estas huancas es probable que formen parte de espacios rituales relacionados a la fertilidad ganadera. La colocación de pagos en el medio de los corrales de animales domésticos hasta la actualidad perdura en muchas comunidades altoandinas e incluso los animales nacidos con deformaciones genéticas es considerado como "illas", hijos de Wamani o de montañas, que son enterrados en el medio de los corrales. Todo esto requiere un estudio minucioso tomando en cuenta los datos etnográficos en los pueblos andinos. 


\section{CONSIDERACiONEs FinAles}

En la cuenca alta del río Huatata, llegamos a identificar un total de 108 monolitos denominados huancas, en los sectores de Ichubamba como también en Yanapiruro. Se caracterizan por ser de formas alargadas, irregulares, lanceoladas, tubulares y rectangulares de distintas longitudes dispuestos verticalmente sobre una pequeña plataforma semicircular. Su asociación cronológica no se pudo determinar por el escaso material cerámico registrado en los alrededores, pero no hay duda que pertenece a las épocas prehispánicas. Según los estudios arqueológicos su presencia es en toda el área andina y han sido registrados en los sitios que pertenecen desde el periodo Precerámico Tardío hasta el Horizonte Tardío. Su funcionalidad según la concepción del mundo andino prehispánico, generalmente está relacionado a la fertilidad de sementeras, protectores de pueblos, dueños de manantiales, protectores de caminantes, entre otros, sin descartar posibles funciones astronómicas.

A partir de las evidencias halladas en el trabajo de campo llegamos a entender que la ubicación espacial de estos monolitos en nuestra área de estudio es el curso superior de los ríos Cayramayu, Rodeohuayqu y Hatunsallahuayqu, afluyentes de río Huatatas, donde se encuentran distribuidas en las crestas y laderas de numerosos colinas. Algunos están asociados directamente a manantiales, otros vinculados a corrales y algunos en zonas infértiles donde abundan pedregales de suelos rocosos. Es posible que estas huancas sean producto de un conjunto de prácticas rituales efectuadas, para el propiciatorio del agua en algún momento de escases o sequia por los pobladores prehispánicas de los valles circundantes. Por otro lado los monolitos ubicados en el centro de los corrales antiguos estarían relacionados a la fertilidad ganadera o algún otro tipo de prácticas rituales. La veracidad de su funcionalidad se encuentra oculta en el corazón de estas numerosas piedras enigmáticas que se conocerá con las futuras investigaciones minuciosas. Somos conscientes de que nuestro trabajo es preliminar y no está sustentado con excavaciones arqueológicas.

\section{Agradecimientos}

Mi sincero reconocimiento a los docentes que me incentivaron para realizar el presente trabajo a Jeffrey Gamarra, Mario Maldonado, Cirilo Vivanco, Ismael Pérez, José Ochatoma y Martha Cabrera. Asimismo expreso mi agradecimiento al arqueólogo Pieter Van Dalen Luna, docentes de la Universidad Nacional Mayor de San Marcos por las sugerencias y recomendaciones para la concretización del presente trabajo. A mis amigos que me apoyaron en el trabajo de campo: Luis Franco Tito, Feliciano Soto Yupanqui, Rosmery López De la Cruz, Yanina Laura Maldonado, Henry Navarro Anyosa e Iván Vivanco Ramos. Finalmente a mis hermanos Abimael y Michael Carhuas por acompañarme en la salida de campo.

\section{REFERENCIAS BiBLIOGRAFÍCAS}

ARRIAGA, Pablo Jose de

1968 (1621) Extirpación de la idolatría del Piru. Biblioteca de Autores Españoles, tomo 209 (Crónicas Peruanas de Interés Indígena), pp. 191-277.Madrid: Ediciones Atlas.

ALBORNOZ, Cristóbal de

1967 (1584) “Instrucción para descubrir todas las guacas del piru y sus camayos y haziendas". Albornoz y el espacio ritual andino prehispánico. Edit. Pierre Duviols Revista Andina 2(1). PP.169-222. 
ÁVILA, Francisco de

1966 (1589?) Dioses hombres de Huarochirí, Narración Quechua recogido por Francisco de Ávila. ed. Bilingüe; traducción de José María Arguedas. Estudio Bibliográfico de Pierre Duviols, Lima.

BAZÁN DEL CAMPO, Francisco

2007 “Las ceremonias especializadas de veneración a las huancas”. Revista arqueológica, SIAN. Año 12 edición instituto SIAN. Trujillo, peru.pp.03-20

CARHUAS TENORIO, Héctor

2015 Prospección arqueológica en la comunidad de Yanapiruro e Ichubamba, Chiara, Ayacucho. Informe de prácticas pre-profesionales, Facultad de Ciencias Sociales de la Universidad Nacional de San Cristóbal de Huamanga.

CAVERO PALOMINO, Yuri I.

2010 Inkapamisan: ushnus y santuario inka en Ayacucho. Ayacucho, Perú.

BAUTISTA GOMEZ, Alfredo Oscar

1993 Prospección arqueológica en Sachabamba, Ayacucho. Informe de prácticas preprofesionales, Facultad de ciencias sociales de la Universidad Nacional de San Cristóbal de Huamanga.

CASAFRANCA, José

1960 "Los nuevos sitios arqueológicos chavinoides en el departamento de Ayacucho". Antiguo Perú: Espacio y Tiempo: 324-334, edit. Juan Mejía Baca, Lima.

\section{CURATOLA PETROCCHI,MarCO}

2008 "La función de los Oráculos en el imperio inca". Adivinación y oráculos en el mundo andino antiguo. Colección de estudios andinos. Lima. pp.15-69.

DE LA CRUZ HUAYTALLA, Rocío

2011 Prospección arqueológica en Quishuarcancha, Chiara -Ayacucho. Informe de prácticas pre-profesionales, Facultad de ciencias sociales de la Universidad Nacional de San Cristóbal de Huamanga.

DUVIOLS, Pierre

1973 "Huari y Llacuaz: agricultores y pastores. Un dualismo prehispánico de oposición y complementariedad”. Revista del Museo Nacional XXXIX. pp. 153-191, Lima.

FALCON HUAYTA, Víctor

2004 "Los orígenes del huanca como objeto de culto en la época pre colonial". Allpanchis,religiones y espacio andino. Instituto de pastoral andina año XXX N ${ }^{\circ} 64$. Segundo semestre.

FARFAN LOBATON, Carlos

2002 “Simbolismo en torno al agua en la comunidad de Huaros, Canta". Boletín de instituto francés de estudios andinos, $N^{\circ} 31(1): 115-142$ 
2012 “El huanca y su dimensión simbólica en la arqueología de la sierra central”. Arqueología y sociedad N 24, Museo de Arqueología y Antropología de UNMSM.

GENTILE, Margarita E.

2003 "Presencia incaica en el paisaje de acontecimientos de un sector de la puna de Juyuy: huanca, usnu, cachauis y quipildor”. Boletín de arqueología PUCP Nº 6. Fondo editorial Pontificia Universidad Católica del Perú, pp.79-106.

GEERTZ, clifford

2003 La interpretación de las culturas. Gedisa, Barcelona.

LEON NINA, Freddy

2010 Impacto ambiental y social en la fase de la construcción del sistema de ductos para el transporte de gas natural del proyecto camisea: Tramo Chiara,Huamanga-Ayacucho, 2006. Pacha Runa $\mathrm{N}^{\circ} 01$, revista del instituto de investigaciones históricas, arqueológicas y antropológicas. Ayacucho, pp.303-324.

LUMBRERAS, Luis Guillermo

1975 Las fundaciones de Huamanga. Hacia una prehistoria de Ayacucho. Homenaje al sesquicentenario de la batalla de Ayacucho. Primera edición. Lima Perú.

MALDONADO VALENZUELA,Mario

2013 "Mito fundacional (llaqta paqariy): construcción de valores sociales en el mundo andino". Alteritas, revista de estudios socioculturales andinos amazónicos. Universidad Nacional de San Cristóbal de Huamanga. Ayacucho, Perú.

ORTEGA HUAYAPA, Edith

2006 Prospección arqueológica en el espacio comprendido entre las quebradas de Cayramayo y Lambrashuayqo, Chiara-Ayacucho. Informe de prácticas pre-profesionales, Facultad de ciencias sociales de la Universidad Nacional de San Cristóbal de Huamanga.

PEASE, Franklin

1973 El Dios Creador Andino. Mosca Azul Ed. Lima.

PÉREZ CALDERÓN Ismael, CRUZ AZAHUANCHE, Noemi y LEÓN NINA, Freddy

2010 Arte rupestre en el valle de Huamanga-Ayacucho. Primera edición. Ayacucho, Perú.

PULGAR VIDAL,Javier

1996 Geografía del Perú, las ocho regiones naturales. Décima edición. PEISA, Lima Perú.

ROCA GOMEZ, Yoni

2007 Reconocimiento arqueológico en Seqchapampa, Chiara Ayacucho. Informe preprofesional. UNSCH. 


\section{ROSTWOROWSKI DE DIEZ CANSECO, María}

1982 Estructuras andinas del poder. Ideología religiosa y política. Instituto de Estudios peruanos. Segunda edición. Lima.

1999 Historia del Tahuantinsuyo. Instituto de Estudios Peruanos. Segunda edición. Lima.

RUIZ ESTRADA, Arturo; HAAS, Jonathan y CREAMER, Winifred.

2011 El mito de vichama y la arqueología en el Norte Chico. GUARA. Revista de museo de arqueología de la Universidad Nacional José Faustino Sánchez Carrión Nº9. pp.7-30

SHADY, Ruth

1997 "La religión como una forma de cohesión social y manejo político en los albores de la civilización en el Perú”. Boletín de Museo de Arqueología y Antropología, Centro C u l t u r a l, Universidad Nacional Mayor de San Marcos. Lima, pp. 13-15

SÁNCHEZ GARCIA, Julio S.

2009 Prospección arqueológica en la quebrada superior de Allpachaca y el río Tambochaca. Informe de prácticas pre-profesionales, Facultad de Ciencias Sociales de la, Universidad Nacional de San Cristóbal de Huamanga, Ayacucho.

2010 Pariahuanca: El misterio de las huancas, estudio de aproximación a la cosmovisión del mundo andino antiguo. Tesis para optar el título de licenciado. Facultad de Ciencias Sociales de la Universidad Nacional de San Cristóbal de Huamanga, Ayacucho.

TELLO ROJAS, Julio Cesar.

1956 Arqueología del valle de Casma. Ed. San Marcos Lima, Perú

VIVANCO, Cirilo y MEDDENS, Frank

2010 “Estudio de usnus del Tawantinsuyo, espacios sagrados en territorios estratégicas de Ayacucho”. Investigacion, Universidad Nacional de San Cristóbal de Huamanga. Vol. 18(1). pp. 81-93. 
